\title{
Adjoint model for estimating material parameters based on microstructure evolution during spinodal decomposition
}

\author{
Yuki Matsuura $\odot,^{*}$ Yuhki Tsukada $\odot,^{\dagger}$ and Toshiyuki Koyama ${ }^{\circ}$ \\ Department of Materials Design Innovation Engineering, Graduate School of Engineering, Nagoya University, \\ Furo-cho, Chikusa-ku, Nagoya 464-8603, Japan
}

(Received 16 June 2021; revised 8 September 2021; accepted 18 October 2021; published 8 November 2021)

\begin{abstract}
Data assimilation techniques are attracting increasing attention because they enable researchers to estimate material parameters by integrating an advanced computational model with experimental data of microstructure evolution. In this study, we develop an adjoint model to integrate a phase-field model for spinodal decomposition with time-series measurement data of compositional field maps to estimate the unknown parameters in the phase-field model. As a case study, simultaneous estimation of six parameters (Gibbs energy parameters, gradient energy coefficient, etc.) in the phase-field model is considered. To confirm the effectiveness of the developed adjoint model, numerical tests called "twin experiments" are conducted using synthetic measurement data prepared in advance through phase-field simulation. In the twin experiments, the optimum estimates of six model parameters of interest are shown to coincide with true values, indicating that several model parameters can be successfully estimated. Furthermore, the effects of the standard deviation of measurement noise $(\sigma)$ and the time interval of measurements $\left(\Delta t_{\text {meas. }}^{\prime}\right)$ on uncertainties of optimum estimates of parameters $\left(\sigma^{\text {est }}\right)$ are examined by conducting twin experiments. It is shown that there is a positive correlation between $\sigma$ and $\sigma^{\text {est }}$ or between $\Delta t_{\text {meas. }}^{\prime}$ and $\sigma^{\text {est }}$, which is essential information for designing experiments to estimate model parameters. The developed adjoint model is assumed to be useful for estimating unknown parameters (e.g., Gibbs energy parameters of a nonequilibrium phase) using time-series measurement data of microstructure evolution during spinodal decomposition.
\end{abstract}

DOI: 10.1103/PhysRevMaterials.5.113801

\section{INTRODUCTION}

Extracting as much information as possible from material microstructure is crucial for accumulating knowledge about the material of interest. Direct comparison between an advanced computational model of microstructure (e.g., a phase-field model [1,2]) and experimental data of microstructure morphology enables researchers to estimate material parameters in the computational model [3]. Machine learning (ML) or data assimilation (DA) techniques are useful for effectively comparing/combining an advanced model with experimental data of microstructure. ML enables researchers to estimate several material parameters $(\boldsymbol{x})$ in a microstructure computational model [4] by exploring a material-parameter region in which the discrepancy $(y)$ between computational results and precipitate shape experimental data becomes small; an efficient exploration can be achieved using a selective sampling strategy based on the Gaussian process modeling of the relation between $x$ and $y$ [5-7]. By contrast, DA is a math-

\footnotetext{
*matsuura.yuki@i.mbox.nagoya-u.ac.jp

†tsukada.yuhki@material.nagoya-u.ac.jp

Published by the American Physical Society under the terms of the Creative Commons Attribution 4.0 International license. Further distribution of this work must maintain attribution to the author(s) and the published article's title, journal citation, and DOI.
}

ematical technique for integrating a simulation model with experimental data, enabling the estimation of model states and parameters and thereby increasing the model's prediction accuracy [8-10]. In "online" DA, a sequential Bayesian filter such as the ensemble Kalman filter (EnKF) is used; measurement data are sequentially obtained in time, and the probability density function (PDF) for model states and parameters is updated using the most recent PDF and newly obtained measurement data. Some efforts have been made to use the EnKF to estimate phase-field model parameters using time-series microstructure data obtained through experiments [11,12] or molecular-dynamics simulation [13]. In "offline" DA, the so-called adjoint method is used, which is known as the four-dimensional variational method in meteorology. Assuming the dynamical model to be perfect, optimum estimates of model states and parameters can be found using previously collected measurement data. In the adjoint method, efficient estimation of model states and parameters is enabled by developing an adjoint model based on the dynamical model. Some adjoint models based on the phase-field model for dendritic crystal growth [14] or grain growth [15] have been proposed for estimating phase-field model parameters using time-series data of microstructure evolution.

Spinodal decomposition in materials has been investigated using various experimental techniques, including transmission electron microscopy (TEM) [16-18], x-ray microtomography [19], x-ray diffraction [16,20], smallangle $\mathrm{x}$-ray scattering [21], and small-angle neutron 
scattering [22]. Aberration-corrected high-angle annular darkfield (HAADF) scanning transmission electron microscopy (STEM) (HAADF-STEM) and electron energy loss spectroscopy enable elemental mapping of microstructure [23,24]. Most recently, a new approach for compositional mapping has been proposed, in which a HAADF-STEM experiment is combined with multislice image simulation results [25]; this provides a way to obtain time-series compositional field maps during spinodal decomposition from HAADF-STEM intensities [26].

Phase-separated structures in glasses such as $\mathrm{BaO}-\mathrm{SiO}_{2}$ [25] and $\mathrm{CaO}-\mathrm{Al}_{2} \mathrm{O}_{3}-\mathrm{SiO}_{2}$ [26] often originate from the spinodal decomposition of an amorphous phase. Gibbs energy parameters of constituent phases are essential for accelerating microstructure optimization through computational approaches such as thermodynamic calculations based on the calculation of phase diagrams method [27] and microstructure simulations based on the phase-field method [1,2]. However, it is inherently difficult to determine the Gibbs energy parameters of an amorphous phase (nonequilibrium phase) through conventional phase equilibrium experiments. As previously mentioned, since time-series data of compositional field maps during spinodal decomposition of an amorphous phase can be obtained experimentally [26], direct comparison between experimental data and an advanced computational model of microstructure evolution should create a new pathway to the estimation of Gibbs energy parameters of an amorphous phase. One of the most promising approaches seems to be the use of a DA technique to integrate experimental data with a phase-field model, which contains unknown parameters (Gibbs energy parameters, etc.) of the amorphous phase of interest. Because a naive implementation of online DA method on high-dimensional problems often results in high computational costs, the offline DA method (adjoint method) seems appropriate for estimating phase-field model states and parameters [15]. Previous studies on the adjoint models based on the phase-field model for dendritic crystal growth or grain growth $[14,15]$ showed that phase-field model parameters, such as interface mobility or grain-boundary mobility, can be successfully estimated using time-series data of the evolution of two-phase microstructure or polycrystalline microstructure. Hence, it is presumed that we can also estimate material parameters using time-series compositional field maps during spinodal decomposition by the adjoint method. However, the adjoint model based on the phase-field model for spinodal decomposition has not been reported and its usefulness in the parameter estimation is unclear.

In this study, we propose an adjoint model to estimate several unknown parameters in a phase-field model for spinodal decomposition using previously collected measurement data of time-series compositional field maps. A two-phase separation has been reported in a wide range of materials [16-26], and a few reports are available on three-phase separation in polymers [28]. Hence, as a case study, a conventional phase-field model for spinodal decomposition in a ternary system is considered, and the corresponding adjoint model is developed. Then, the effectiveness of the developed adjoint model is confirmed using synthetic measurement data prepared in advance through phase-field simulation; this numerical test of the newly developed model is known as a twin experiment. In Sec. II, we explain a phase-field model, adjoint model, simulation conditions, and twin experiments. In Sec. III, twin experiments are performed by varying initial guesses of parameters of interest, the measurement noise, and the time interval of measurements to clarify problems in parameter estimation and to gain insight into the effects of measurement conditions on the optimum estimates of parameters. Section IV discusses the results, and Sec. V summarizes the main conclusions.

\section{CALCULATION METHOD}

\section{A. Phase-field model for spinodal decomposition in a ternary system}

The spinodal decomposition of a nonequilibrium phase in a virtual A-B-C ternary system is modeled. The concentration of component $X(X=\mathrm{A}, \mathrm{B}, \mathrm{C}), c_{X}(\mathbf{r}, t)$, is used as a field variable. Hereafter, $c_{\mathrm{A}}, c_{\mathrm{B}}$, and $c_{\mathrm{C}}$ are denoted as $c_{1}, c_{2}$, and $c_{3}$, respectively. $c_{2}$ and $c_{3}$ are assigned to independent variables, and their temporal evolution is given by the Cahn-Hilliard equation [1]:

$$
\frac{\partial c_{i}}{\partial t}=\nabla \cdot\left(\sum_{j=2}^{3} M_{i j} \nabla \frac{\delta G_{\text {sys }}}{\delta c_{j}}\right), \quad i=2,3,
$$

where $G_{\text {sys }}$ is the total free energy of the system and $M_{i j}$ is the Onsager coefficient. $G_{\text {sys }}$ is a functional of the field variables and is defined as

$$
G_{\mathrm{sys}}=\int_{\mathbf{r}}\left(G_{\mathrm{c}}+\frac{1}{2} \sum_{i=1}^{3} \kappa_{i}\left(\nabla c_{i}\right)^{2}\right) d \mathbf{r},
$$

where $G_{\mathrm{c}}$ represents the Gibbs energy of the phase and $\kappa_{i}$ represents the gradient energy coefficient [29]. $G_{\mathrm{c}}$ is formulated on the basis of the subregular solution model [27]:

$$
\begin{aligned}
G_{\mathrm{c}}= & c_{1}{ }^{0} G_{\mathrm{A}}+c_{2}{ }^{0} G_{\mathrm{B}}+c_{3}{ }^{0} G_{\mathrm{C}}+\mathrm{R} T \sum_{i=1}^{3} c_{i} \ln c_{i} \\
& +c_{1} c_{2} L_{\mathrm{A}, \mathrm{B}}+c_{1} c_{3} L_{\mathrm{A}, \mathrm{C}}+c_{2} c_{3} L_{\mathrm{B}, \mathrm{C}},
\end{aligned}
$$

where ${ }^{0} G_{i}$ represents the Gibbs energy of the phase containing the pure component $i, \mathrm{R}$ the gas constant, $T$ the absolute temperature, and $L_{i, j}$ the interaction parameter. We consider the case in which $L_{\mathrm{A}, \mathrm{B}}$ is composition independent, whereas $L_{\mathrm{A}, \mathrm{C}}$ and $L_{\mathrm{B}, \mathrm{C}}$ are composition dependent:

$$
\begin{aligned}
L_{\mathrm{A}, \mathrm{C}} & ={ }^{0} L_{\mathrm{A}, \mathrm{C}}+{ }^{1} L_{\mathrm{A}, \mathrm{C}}\left(c_{1}-c_{3}\right) \\
& ={ }^{0} L_{\mathrm{A}, \mathrm{C}}+{ }^{1} L_{\mathrm{A}, \mathrm{C}}\left(1-c_{2}-2 c_{3}\right), \\
L_{\mathrm{B}, \mathrm{C}} & ={ }^{0} L_{\mathrm{B}, \mathrm{C}}+{ }^{1} L_{\mathrm{B}, \mathrm{C}}\left(c_{2}-c_{3}\right) .
\end{aligned}
$$

For simplicity, we assume that $M_{i j}$ is composition independent, ${ }^{0} G_{\mathrm{A}}={ }^{0} G_{\mathrm{B}}={ }^{0} G_{\mathrm{C}}=0$, and $\kappa_{i}=\kappa$. Refer to Appendix A for the derivation of Eq. (1).

\section{B. Adjoint model}

Given the time-series measurement data of compositional field maps, we develop an adjoint model for estimating six time-invariant parameters, ${ }^{0} L_{\mathrm{A}, \mathrm{C}},{ }^{1} L_{\mathrm{A}, \mathrm{C}},{ }^{0} L_{\mathrm{B}, \mathrm{C}},{ }^{1} L_{\mathrm{B}, \mathrm{C}}, \kappa$, 
and $M_{22}$ in the phase-field model. The state vector $\mathbf{x}(t)$ is expressed as

$$
\begin{gathered}
\mathbf{x}(t)=\left(\mathbf{c}(t)^{\mathrm{T}}, \mathbf{p}(t)^{\mathrm{T}}\right)^{\mathrm{T}}, \\
\mathbf{c}(t)=\left(c_{2}^{(1)}(t), c_{2}^{(2)}(t), \cdots, c_{2}^{\left(N_{\text {grid }}\right)}(t), c_{3}^{(1)}(t),\right. \\
\left.\left.c_{3}^{(2)}(t), \cdots, c_{3}^{\left(N_{\text {grid }}\right)}(t)\right)\right)^{\mathrm{T}}, \\
\mathbf{p}(t)=\left({ }^{0} L_{\mathrm{A}, \mathrm{C}}(t),{ }^{1} L_{\mathrm{A}, \mathrm{C}}(t),{ }^{0} L_{\mathrm{B}, \mathrm{C}}(t),\right. \\
\left.{ }^{1} L_{\mathrm{B}, \mathrm{C}}(t), \kappa(t), M_{22}(t)\right)^{\mathrm{T}},
\end{gathered}
$$

where $\bullet$ T denotes the transpose of $\bullet, N_{\text {grid }}$ is the number of computational grid points, $c_{X}^{(n)}(t)$ is the concentration of component $X$ on a computational grid point $n(n=$ $\left.1,2, \ldots, N_{\text {grid }}\right)$ at time $t$. Note that $\mathbf{p}(t)=\mathbf{p}(0)$, and $\mathbf{c}(t) \in$ $\mathbb{R}^{2 N_{\text {grid }}}, \mathbf{p}(t) \in \mathbb{R}^{6}, \mathbf{x}(t) \in \mathbb{R}^{2 N_{\text {grid }}+6}$. The dynamical model is expressed as

$$
\frac{\partial \mathbf{x}}{\partial t}=\mathbf{F}(\mathbf{x}),
$$

which corresponds to Eq. (1) for $\mathbf{c}$, and $\partial \mathbf{p} / \partial t=0$ for $\mathbf{p}$. We assume that the experimental measurement points of composition are coincident with the computational grid points; thus, the dimension of measurement data at an instance is equal to that of $\mathbf{c}$. The measurement $c_{X_{-} \text {meas. }}^{(n)}$ is modeled by

$$
c_{X \_ \text {meas. }}^{(n)}(t)=c_{X}^{(n)}(t)+v^{(n)}(t),
$$

where $v^{(n)}(t)$ is a white-noise term with mean zero and variance $\sigma^{2}$. A cost function is defined as

$$
\begin{gathered}
J=\int_{0}^{t_{\max }} \mathcal{J} d t \\
\mathcal{J}=\frac{1}{2 \sigma^{2}} \sum_{m=1}^{N_{\text {meas. }}} \delta\left(t-t_{m}\right) \sum_{i=2}^{3} \sum_{n=1}^{N_{\text {grid }}}\left(c_{i \_ \text {meas. }}^{(n)}(t)-c_{i}^{(n)}(t)\right)^{2},
\end{gathered}
$$

where $t_{m}\left(m=1,2, \ldots, N_{\text {meas. }}\right)$ is the measurement location in time, $N_{\text {meas. }}$ represents the number of measurements, and $\delta(\bullet)$ represents the Dirac delta function [14]. The problem is to find the optimum solution $\hat{\mathbf{p}}(0)$ that minimizes $J$ under the constraint that the dynamical model [Eq. (9)] is perfect. Here the Lagrangian is introduced:

$$
\mathcal{L}=\int_{0}^{t_{\max }} \mathcal{J} d t+\int_{0}^{t_{\max }} \lambda^{\mathrm{T}}\left(\mathbf{F}(\mathbf{x})-\frac{\partial \mathbf{x}}{\partial t}\right) d t,
$$

where $\lambda(t) \in \mathbb{R}^{2 N_{\text {grid }}+6}$ is the Lagrangian multiplier vector. Calculating the variational derivative of $\mathcal{L}$, the temporal evolution of $\boldsymbol{\lambda}$ is given by the adjoint model:

$$
\begin{gathered}
-\frac{\partial \lambda}{\partial t}=\left(\frac{\partial \mathbf{F}}{\partial \mathbf{x}}\right)^{\mathrm{T}} \lambda+\frac{\partial \mathcal{J}}{\partial \mathbf{x}}, \\
\lambda\left(t_{\max }\right)=\mathbf{0}, \\
\lambda(0)=\frac{\partial J}{\partial \mathbf{x}(0)} .
\end{gathered}
$$

Refer to Appendix B for the final form of Eq. (14). In the iterative framework to find $\hat{\mathbf{p}}(0)$, the following procedure is used:

Iteration: $k=0,1,2, \cdots$.
TABLE I. Simulation parameters. The symbol with a prime denotes the dimensionless quantity in numerical analysis.

\begin{tabular}{ll}
\hline \hline Temperature $(\mathrm{K})$ & $T=900$ \\
Unit time step & $\Delta t^{\prime}=0.005$ \\
Unit grid size $(\mathrm{nm})$ & $l_{0}=1$ \\
Onsager coefficient & $M_{22}^{\prime}=M_{33}^{\prime}=1.0$ \\
& $M_{23}^{\prime}=M_{32}^{\prime}=-0.5$ \\
Interaction parameter $\left(\mathrm{J} \mathrm{mol}^{-1}\right)$ & $L_{\mathrm{A}, \mathrm{B}}=25000$ \\
& ${ }^{0} L_{\mathrm{A}, \mathrm{C}}=22000,{ }^{1} L_{\mathrm{A}, \mathrm{C}}=-3000$ \\
& ${ }^{0} L_{\mathrm{B}, \mathrm{C}}=25000,{ }^{1} L_{\mathrm{B}, \mathrm{C}}=0$ \\
Gradient energy coefficient & $\kappa=5.0 \times 10^{-15}$ \\
$\quad$ & \\
\hline \hline
\end{tabular}

Step 1: Starting with $\mathbf{x}_{k}(0)$, solve the forward model [Eq. (9)] for $\mathbf{x}$.

Step 2: Using the given set of measurement data $c_{X \text { meas. }}^{(n)}$, calculate $\partial \mathcal{J} / \partial \mathbf{x}$.

Step 3: Starting with $\lambda\left(t_{\max }\right)=\mathbf{0}$, solve the backward model [Eq. (14)] for $\lambda$ to obtain $\lambda(0)=\partial J / \partial \mathbf{x}_{k}(0)$.

Step 4: The obtained $\partial J / \partial \mathbf{x}_{k}(0)$ is used to compute $\mathbf{x}_{k+1}(0)$. If $\left\|\mathbf{x}_{k+1}-\mathbf{x}_{k}\right\|<\varepsilon$, stop. Else go to step 1 .

$\mathbf{x}_{k+1}(0)$ is computed on the basis of the quasi-Newton method; Hessian is estimated using the Broyden-FletcherGoldfarb-Shanno method [30]. The step length is computed on the basis of Armijo's rule [31]. The tolerance is set to $\varepsilon=10^{-15}$

\section{Simulation conditions}

Two-dimensional simulations are performed using $100 \times$ 100 computational grids $\left(N_{\text {grid }}=10000\right)$. A periodic boundary condition is assumed. Equations (1) and (14) are solved using the conventional finite-difference method with the explicit Euler technique. In the numerical analysis, all physical parameters are reduced to dimensionless quantities using the following scaling factors: the product of the gas constant and the absolute temperature, $\mathrm{R} T$, for energy; the unit grid size in the difference method, $l_{0}$, for length; and $\left(M_{33} \mathrm{R} T / l_{0}^{2}\right)^{-1}$ for time. The parameters used in the simulations are summarized in Table I.

Figure 1(a) shows the A-B-C ternary phase diagram at $900 \mathrm{~K}$ calculated using the interaction parameters shown in Table I. The black dot denotes the alloy composition, $c_{1}=$ $c_{2}=c_{3}=1 / 3$, which is located within the three-phase region. Figure 1(b) shows the results of phase-field simulation using the parameters shown in Table I. The initial microstructure at $t^{\prime}=0$ was prepared in advance using a phase-field simulation, which was performed for 2000 time steps starting from the single phase with compositional fluctuation of $\pm 1 \%$ from the alloy composition. We see from Fig. 1(b) that the phase separation progresses in two stages; the single phase first separates into B-rich and B-lean phases $\left(t^{\prime}=0-30\right)$, and subsequently, the B-lean phase separates into A-rich and C-rich phases $\left(t^{\prime}=30-50\right)$.

\section{Twin experiment}

To confirm the effectiveness of the developed adjoint model, twin experiments are performed as follows: 

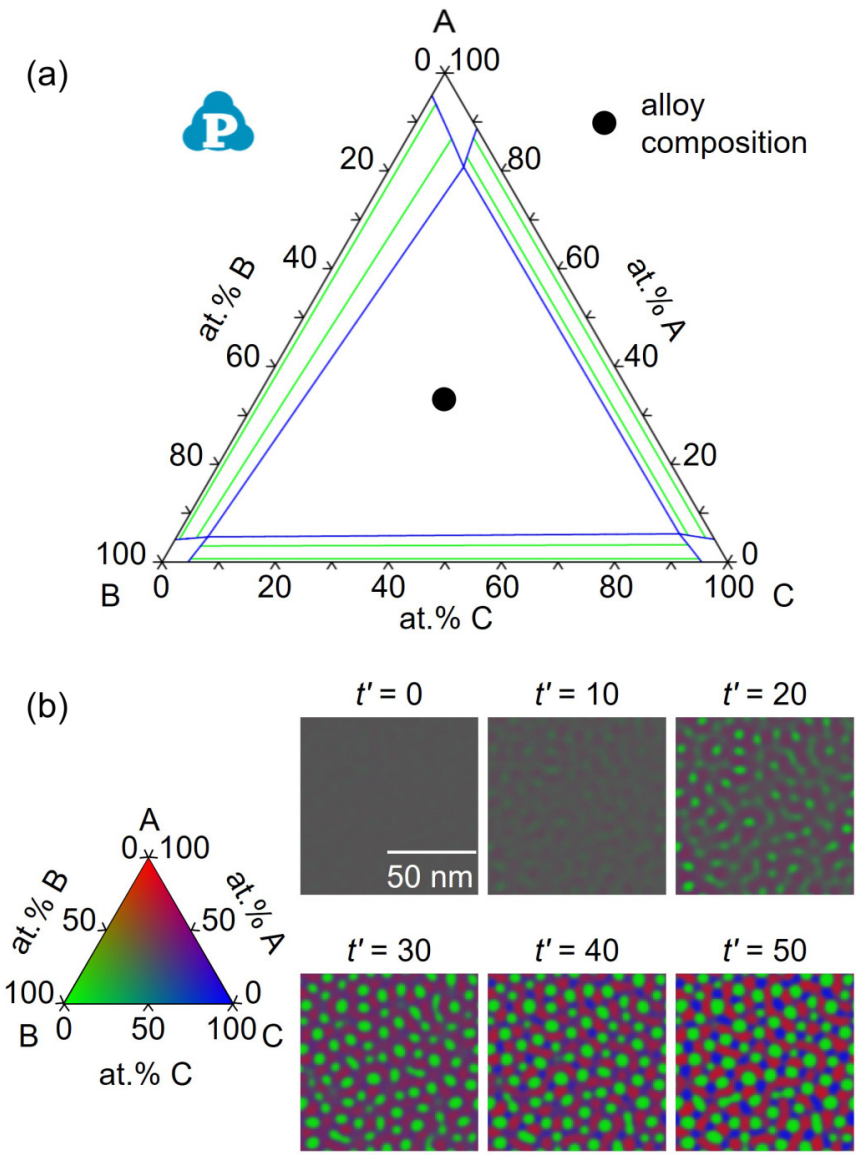

FIG. 1. Calculation result of phase diagram at $900 \mathrm{~K}$ in the A-B-C ternary system (a) and phase-field simulation results of microstructure evolution (b), both of which are obtained using the parameters shown in Table I. The ternary color legend is used to show the ternary compositional field in (b). $t^{\prime}$ denotes the dimensionless time in numerical analysis.

Step 1: Assign true values to the elements of $\mathbf{p}(0)=$ $\left({ }^{0} L_{\mathrm{A}, \mathrm{C}},{ }^{1} L_{\mathrm{A}, \mathrm{C}},{ }^{0} L_{\mathrm{B}, \mathrm{C}},{ }^{1} L_{\mathrm{B}, \mathrm{C}}, \kappa, M_{22}\right)^{\mathrm{T}}$.

Step 2: Using the true vector $\mathbf{p}^{\text {true }}(0)$, solve the forward model [Eq. (1)] to compute $\mathbf{c}(t)=\left(c_{2}^{(1)}(t)\right.$, $\left.c_{2}^{(2)}(t), \cdots, c_{2}^{\left(N_{\text {grid }}\right)}(t), c_{3}^{(1)}(t), c_{3}^{(2)}(t), \cdots, c_{3}^{\left(N_{\text {grid }}\right)}(t)\right)^{\mathrm{T}}$.

Step 3: Using $\mathbf{c}(t)$ computed in Step 2, generate the synthetic measurement data by $c_{X_{-} \text {meas. }}^{(n)}(t)=c_{X}^{(n)}(t)+$ $v^{(n)}(t)$, where $v^{(n)}(t)$ is a white-noise term with mean zero and variance $\sigma^{2}$.

Step 4: Integrate the measurement data $c_{X_{-} \text {meas. }}^{(n)}(t)$ with the phase-field model to estimate the optimum solution $\hat{\mathbf{p}}(0)$ using the iterative procedure described in Sec. II B.

The values of ${ }^{0} L_{\mathrm{A}, \mathrm{C}},{ }^{1} L_{\mathrm{A}, \mathrm{C}},{ }^{0} L_{\mathrm{B}, \mathrm{C}},{ }^{1} L_{\mathrm{B}, \mathrm{C}}, \kappa$, and $M_{22}$ shown in Table I are assumed as the true values of the elements of $\mathbf{p}(0)$. Hence, the synthetic measurement data are generated by adding the noise term $v^{(n)}(t)$ to the compositional fields shown in Fig. 1(b).

In Sec. III, three types of twin experiments (I, II, and III) are performed. In each test in the twin experiments, measurements are assumed to be obtained at an equal time interval $\Delta t_{\text {meas. }}^{\prime}$, and the number of measurements is given by $N_{\text {meas. }}=t_{\text {max }}^{\prime} / \Delta t_{\text {meas. }}^{\prime}$. Initial guesses of the elements of $\mathbf{p}(0)$ used in the twin experiments are summarized in Table II. Twin experiment $\mathrm{I}$ is conducted to determine whether the optimum vector $\hat{\mathbf{p}}(0)$ estimated using the adjoint model coincides with the true vector $\mathbf{p}^{\text {true }}(0)$. Note that in twin experiment $I$, the noise term $v^{(n)}(t)$ is ignored in generating the synthetic measurement data for simplicity. Twin experiment II is conducted to examine how the magnitude of variance $\sigma^{2}$ in the measurement noise affects the elements of $\hat{\mathbf{p}}(0)$ (optimum estimates of parameters) and their uncertainties. Twin experiment III is conducted to examine the effect of the time interval of measurements $\Delta t_{\text {meas. }}^{\prime}$ on the optimum estimates of parameters and their uncertainties. In twin experiments II and III, 100 tests are conducted for each condition of $\sigma^{2}$ and $\Delta t_{\text {meas. }}^{\prime}$ using 100 synthetic measurement data with different noise terms generated from different seeds of pseudorandom numbers.

\section{RESULTS}

\section{A. Twin experiment I: Effect of initial guess}

To determine whether $\hat{\mathbf{p}}(0)$ coincides with $\mathbf{p}^{\text {true }}(0)$, twin experiment I was conducted under $\Delta t_{\text {meas. }}^{\prime}=10\left(N_{\text {meas. }}=5\right)$ for three sets of initial guesses [I-(i), I-(ii), and I-(iii)] of the elements of $\mathbf{p}(0)$ shown in Table II. Figure 2 illustrates the results of the twin experiment using the initial guess I-(i). Figure 2(a) illustrates the A-B-C ternary phase diagram at 900 $\mathrm{K}$ calculated with the initial guess I-(i). The alloy composition indicated by the black dot in Fig. 2(a) is located within the three-phase region. Figure 2(b) depicts the change in the cost function $J$ as the number of iterations $k$ for updating $\mathbf{p}(0)$ is increased; the value of $J$ decreases as $k$ increases. The changes in the estimates of six parameters [elements of $\mathbf{p}(0)$ ] as $k$ increases are shown in Figs. 2(c)-2(f), where the true values are indicated by dotted lines. The values of all six parameters approach the true values at $k=15$ and finally converge to the true values at $k=30$. This result shows that the developed adjoint model is effective to estimate several material parameters simultaneously based on the time-series measurement data of compositional field maps. In the twin experiment using the initial guess I-(ii), we also confirmed that all optimum estimates of six parameters were coincident with the true values (see Supplemental Material Fig. S1 [32]).

Figure 2(b) shows that the value of $J$ decreases in two stages: $k=0-6$ and $k=6-30$. A similar result was also confirmed in the twin experiment using the initial guess I-(ii) (see Supplemental Material Fig. S1 [32]). Figure 3(b) depicts the phase-field simulation results using the estimates of parameters at $k=0,4,8$, and 30 . In the simulation with the estimates of parameters at $k=0$ (initial guesses), spinodal decomposition does not occur, and hence, the simulation results significantly differ from the synthetic measurement data [Fig. 1(b)]. This is attributable to the initial guess of the Onsager coefficient $M_{22}$, whose value is smaller than the true one [see the initial guess I-(i) in Table II]. In the simulation with the estimates of parameters at $k=4$, the single phase separates into B-rich and B-lean phases, but the subsequent phase separation does not sufficiently occur. Although the 
TABLE II. True values and initial guesses of six parameters of interest in twin experiments. True values are used to generate the synthetic measurement data. The symbol with a prime denotes the dimensionless quantity in numerical analysis.

\begin{tabular}{|c|c|c|c|c|c|c|}
\hline & $\begin{array}{c}{ }^{0} L_{\mathrm{A}, \mathrm{C}} \\
\left(\mathrm{J} \mathrm{mol}^{-1}\right)\end{array}$ & $\begin{array}{c}{ }^{1} L_{\mathrm{A}, \mathrm{C}} \\
\left(\mathrm{J} \mathrm{mol}^{-1}\right)\end{array}$ & $\begin{array}{c}{ }^{0} L_{\mathrm{B}, \mathrm{C}} \\
\left(\mathrm{J} \mathrm{mol}^{-1}\right)\end{array}$ & $\begin{array}{c}{ }^{1} L_{\mathrm{B}, \mathrm{C}} \\
\left(\mathrm{J} \mathrm{mol}^{-1}\right)\end{array}$ & $\begin{array}{c}\kappa \\
\left(\mathrm{J} \mathrm{m}^{2} \mathrm{~mol}^{-1}\right)\end{array}$ & $M_{22}^{\prime}$ \\
\hline True value & 22000 & -3000 & 25000 & 0 & $5.0 \times 10^{-15}$ & 1.0 \\
\hline Initial guess I-(i) & 20000 & 0 & 20000 & 0 & $6.0 \times 10^{-15}$ & 0.6 \\
\hline Initial guess I-(ii) & 25500 & -4000 & 25500 & -4000 & $8.0 \times 10^{-15}$ & 0.9 \\
\hline Initial guess I-(iii) & 17000 & -3000 & 25000 & 0 & $3.0 \times 10^{-15}$ & 1.3 \\
\hline Initial guess II & 25500 & -4000 & 25500 & -4000 & $8.0 \times 10^{-15}$ & 0.9 \\
\hline Initial guess III & 20000 & 0 & 20000 & 0 & $6.0 \times 10^{-15}$ & 0.6 \\
\hline
\end{tabular}

value of $J$ decreases as $k$ increases, the simulation results with the estimates of parameters at $k=4$ still significantly differ from the synthetic measurement data [Fig. 1(b)], where threephase spinodal decomposition is observed. In the simulations with the estimates of parameters at $k=8$ and 30 , the single phase separates into B-rich and B-lean phases, followed by the separation of the B-lean phase into the A-rich and C-rich phases. The three-phase microstructure simulated using the (a)

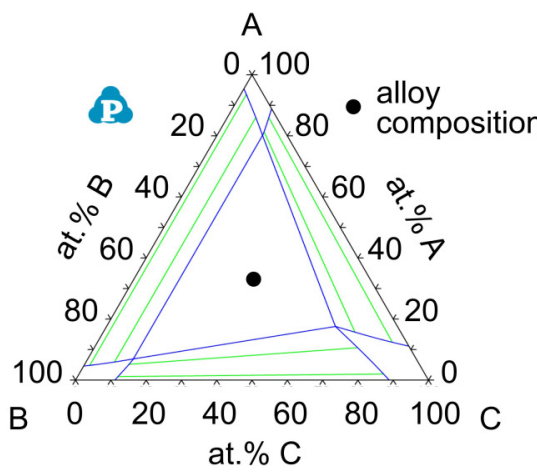

(c)

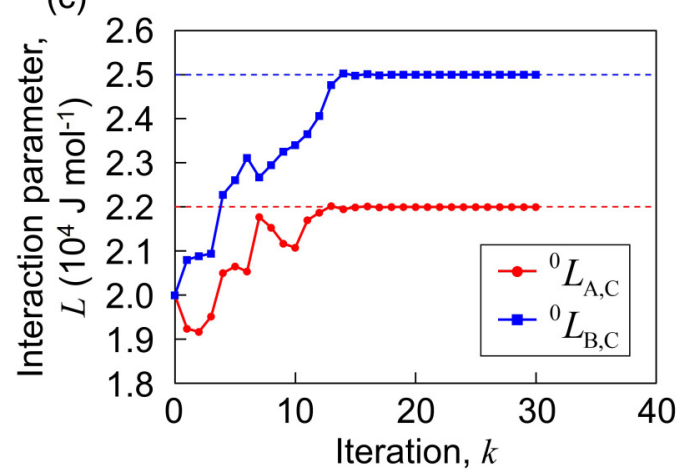

(e)

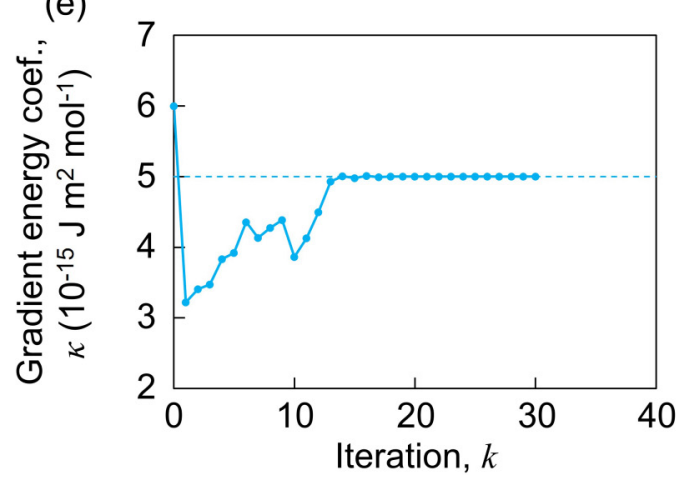

(b)

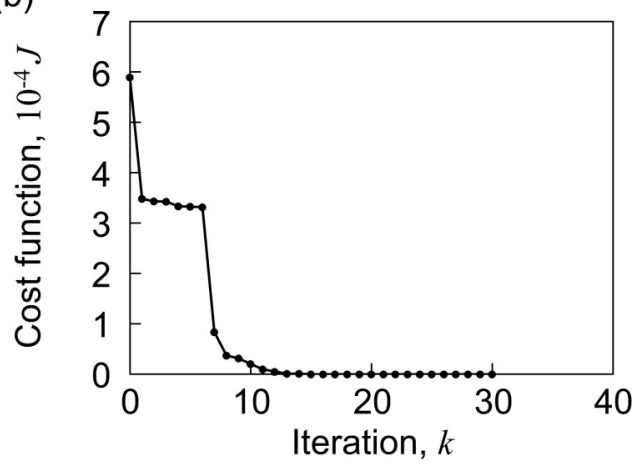

(d)

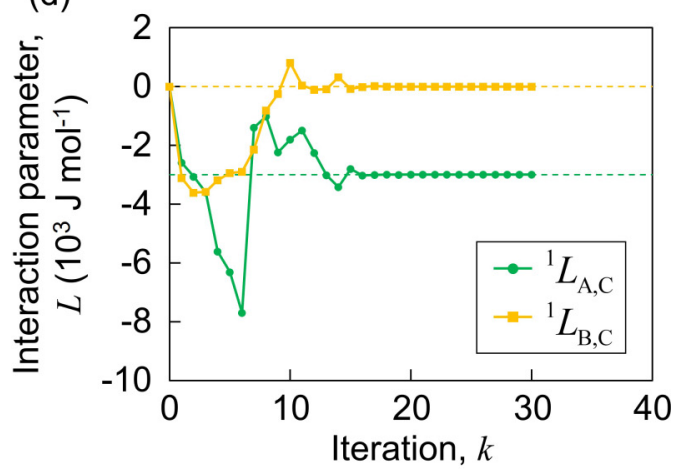

(f)

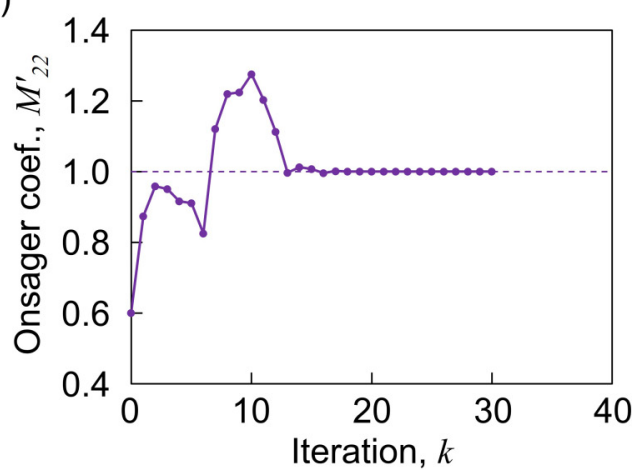

FIG. 2. Results of twin experiment using the initial guess I-(i) shown in Table II: (a) phase diagram at $900 \mathrm{~K}$ in the A-B-C ternary system calculated using the initial guess I-(i), (b) change in the cost function $J$ as the number of iterations $k$ increases, and (c)-(f) changes in the estimates of six parameters with increasing $k$. True values of the six parameters are indicated by dotted lines in (c)-(f). 
(a)

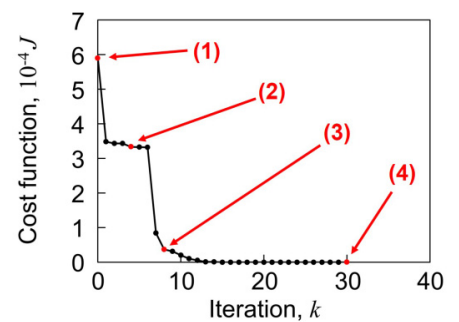

(b)

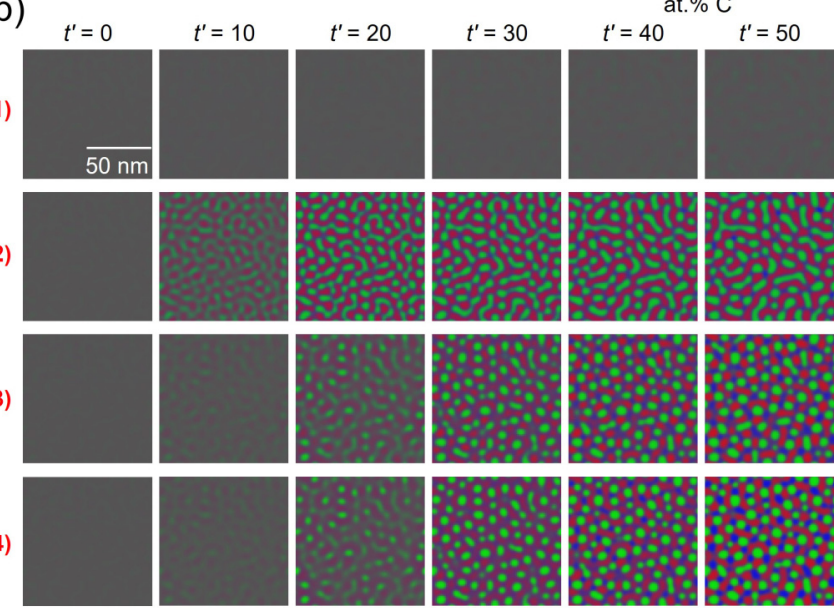

FIG. 3. Change in the cost function $J$ as the number of iterations $k$ increases in twin experiment I-(i) (Fig. 2) (a) and results of phasefield simulations using the estimates of six parameters at (1) $k=0$, (2) $k=4$, (3) $k=8$, and (4) $k=30$ (b). The ternary color legend is used to show the ternary compositional field in (b). $t^{\prime}$ denotes the dimensionless time in numerical analysis.

optimum estimates of parameters at $k=30$ coincides with the synthetic measurement data [Fig. 1(b)], and the value of $J$ becomes approximately zero.

Figure 4 shows the results of twin experiment I using the initial guess I-(iii) shown in Table II. Figure 4(a) depicts the A-B-C ternary phase diagram at $900 \mathrm{~K}$ calculated using the initial guess I-(iii). The alloy composition indicated by the black dot in Fig. 4(a) is located within the three-phase region. Figure 4(b) illustrates how the value of $J$ changes as the number of iterations $k$ increases, and the corresponding changes in the estimates of six parameters are shown in Figs. 4(c)-4(f). At $k=36$, all values of six parameters converge to certain values that are different from the true ones. This result indicates that there would be some local minima of $J$ in multidimensional parameter space, which could lead to parameter estimation failure, depending on the initial guesses of parameters. Figures 5(a) and 5(b) show the results of phase diagram calculation and phase-field simulation, respectively, both of which are obtained using the optimum estimates of parameters at $k=36$ in Fig. 4. The alloy composition is located within the two-phase region in the A-B-C ternary phase diagram [Fig. 5(a)], and a phase separation into B-rich and B-lean phases is observed in the phase-field simulation [Fig. 5(b)]. As we already know from the synthetic measurement data [Fig. 1(b)] that the alloy composition should be located in the three-phase region in the A-B-C ternary phase
TABLE III. Measurement conditions in twin experiments II and III. The symbol with a prime denotes the dimensionless quantity in numerical analysis.

\begin{tabular}{lcccc}
\hline \hline & $\sigma$ & $\Delta t_{\text {meas. }}^{\prime}$ & $N_{\text {meas. }}$ & $t_{\text {max }}^{\prime}$ \\
\hline Twin experiment II & $1 \times 10^{-5}-5 \times 10^{-2}$ & 10 & 5 & 50 \\
Twin experiment III & $1 \times 10^{-2}$ & $0.5-50$ & $1-100$ & 50 \\
\hline \hline
\end{tabular}

diagram, it is clear from Fig. 5(a) that the optimum estimates of interaction parameters ${ }^{0} L_{\mathrm{A}, \mathrm{C}},{ }^{1} L_{\mathrm{A}, \mathrm{C}},{ }^{0} L_{\mathrm{B}, \mathrm{C}},{ }^{1} L_{\mathrm{B}, \mathrm{C}}$ are incorrect. In other words, the phase diagram calculation using the optimum estimates of ${ }^{0} L_{\mathrm{A}, \mathrm{C}},{ }^{1} L_{\mathrm{A}, \mathrm{C}},{ }^{0} L_{\mathrm{B}, \mathrm{C}},{ }^{1} L_{\mathrm{B}, \mathrm{C}}$ is useful as a preliminary screening method for confirming the validity of their values. In addition, the apparent discrepancy between a phase-field simulation using the optimum estimates of parameters [Fig. 5(b)] and the synthetic measurement data [Fig. 1(b)] shows that the parameter estimation failed.

The success rate of the parameter estimation was evaluated using multiple sets of initial guesses of six parameters, whose values were set close to the true values as ${ }^{0} L_{\mathrm{A}, \mathrm{C}}=22000 \pm$ $1000\left(\mathrm{~J} \mathrm{~mol}^{-1}\right),{ }^{1} L_{\mathrm{A}, \mathrm{C}}=-3000 \pm 1000\left(\mathrm{~J} \mathrm{~mol}^{-1}\right),{ }^{0} L_{\mathrm{B}, \mathrm{C}}=$ $25000 \pm 1000\left(\mathrm{~J} \mathrm{~mol}^{-1}\right),{ }^{1} L_{\mathrm{B}, \mathrm{C}}= \pm 1000\left(\mathrm{~J} \mathrm{~mol}^{-1}\right), \kappa=$ $5.0 \times 10^{-15} \pm 0.5 \times 10^{-15}\left(\mathrm{~J} \mathrm{~m}^{2} \mathrm{~mol}^{-1}\right)$, and $M_{22}^{\prime}=1.0 \pm$ 0.05 . From the results of $64\left(=2^{6}\right)$ twin experiments using 64 sets of initial parameter guesses, the success rate of the parameter estimation was computed as $90.6 \%(=58 / 64)$. Overall, the results of twin experiment I indicate that the estimation of model parameters should be performed several times by varying initial guesses of parameters, and the validity of the estimation results needs to be assessed on the basis of the values of $J$, phase diagram calculations, and phase-field simulations using the optimum estimates of parameters.

\section{B. Twin experiment II: Effect of measurement noise}

Twin experiment II was conducted to examine the effect of the magnitude of variance $\sigma^{2}$ in the measurement noise on the optimum estimates of parameters and their uncertainties. The initial guesses of six parameters [elements of $\mathbf{p}(0)]$ and the measurement condition are shown in Tables II and III, respectively. Twin experiments were conducted by varying the magnitude of $\sigma$; for each condition of $\sigma, 100$ twin experiments were conducted using 100 synthetic measurement data with different noise terms generated from different seeds of pseudorandom numbers. Figure 6 shows the mean values and standard deviations $\left(\sigma^{\text {est }}\right)$ of optimum estimates of six parameters under various conditions of $\sigma$. Six parameters are successfully estimated under any conditions of $\sigma$. The relation between $\sigma$ and $\sigma^{\text {est }}$ for each parameter is shown in Fig. 7, with a positive correlation between $\sigma$ and $\sigma^{\text {est }}$. The results of Figs. 6 and 7 indicate that the requirement for estimating all six parameters with three significant digits is $\sigma \leqslant 1 \times 10^{-2}$.

\section{Twin experiment III: Effect of time interval of measurements}

Twin experiment III was conducted to examine the effect of the time interval of measurements $\left(\Delta t_{\text {meas. }}^{\prime}\right)$ on the optimum estimates of parameters and their uncertainties. The 
(a)

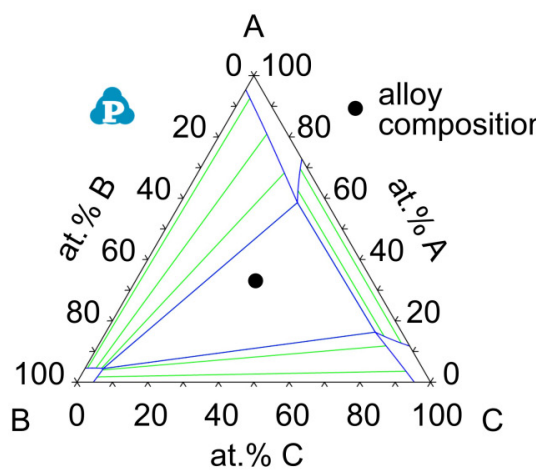

(c)

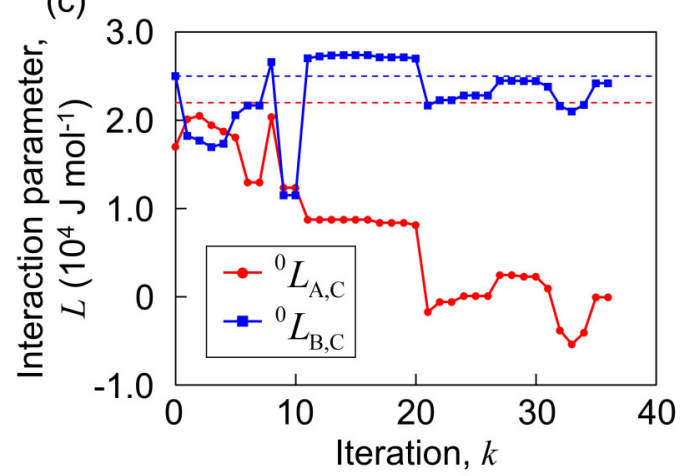

(e)

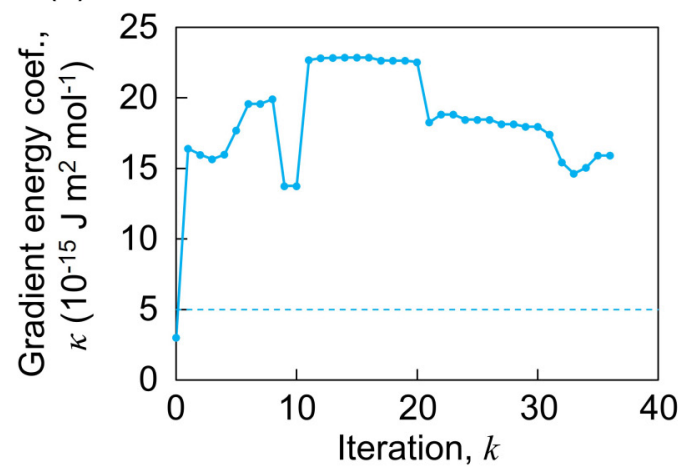

(b)

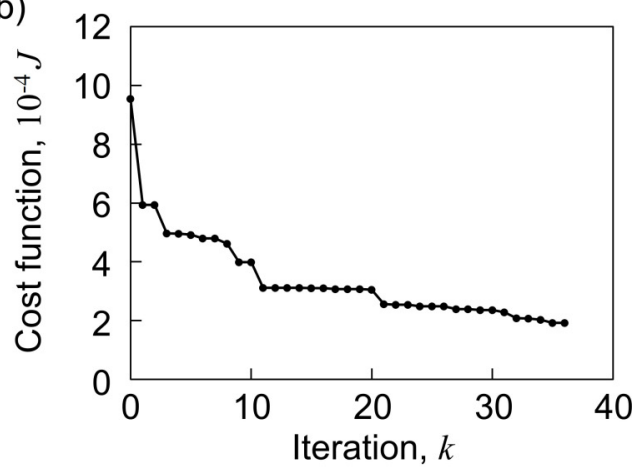

(d)

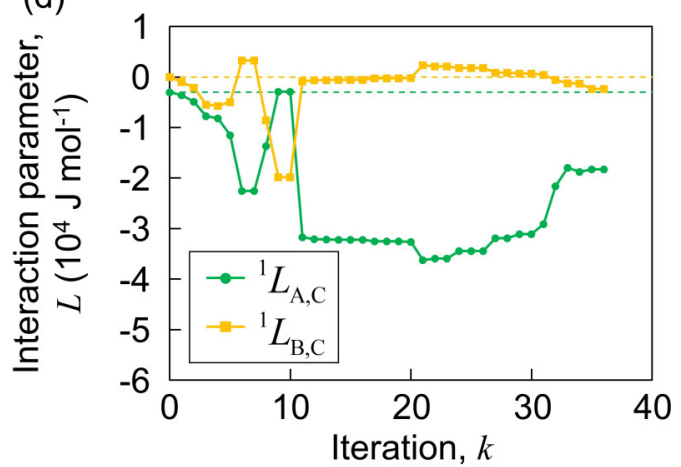

(f)

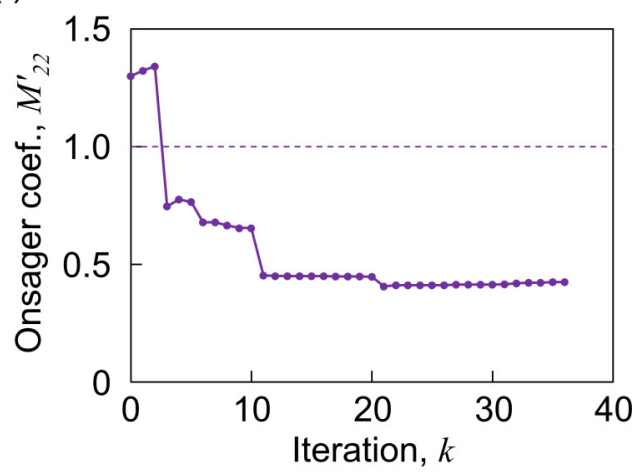

FIG. 4. Results of twin experiment using the initial guess I-(iii) shown in Table II: (a) phase diagram at $900 \mathrm{~K}$ in the A-B-C ternary system calculated using the initial guess I-(iii), (b) change in the cost function $J$ as the number of iterations $k$ increases, and (c)-(f) changes in the estimates of six parameters with increasing $k$. True values of the six parameters are indicated by dotted lines in (c)-(f).

initial guesses of six parameters [elements of $\mathbf{p}(0)$ ] and the measurement condition are shown in Tables II and III, respectively. Twin experiments were conducted by varying $\Delta t_{\text {meas. }}^{\prime}$ and thereby varying the number of measurements $N_{\text {meas. }}=t_{\text {max }}^{\prime} / \Delta t_{\text {meas. }}^{\prime}$; for each condition of $\Delta t_{\text {meas. }}^{\prime}, 100$ twin experiments were conducted using 100 synthetic measurement data with different noise terms generated from different seeds of pseudorandom numbers. Figure 8 depicts the mean values and standard deviations $\left(\sigma^{\text {est }}\right)$ of optimum estimates of six parameters under various conditions of $\Delta t_{\text {meas }}^{\prime}$. It is seen that all six parameters are successfully estimated under any conditions except for $\Delta t_{\text {meas. }}^{\prime}=5$ (or $N_{\text {meas. }}=10$ ). Under $\Delta t_{\text {meas. }}^{\prime}=5$, the mean values of optimum estimates of parameters significantly differ from the true values, and $\sigma^{\text {est }}$ is noticeably larger than it would be under other conditions of $\Delta t_{\text {meas. }}^{\prime}$. The relation between $\Delta t_{\text {meas. }}^{\prime}$ and $\sigma^{\text {est }}$ is shown in Fig. 9; there is a positive correlation between $\Delta t_{\text {meas. }}^{\prime}$ and $\sigma^{\text {est }}$ if the results for $\Delta t_{\text {meas. }}^{\prime}=5$ are excluded.
The 100 sets of optimum estimates of parameters under $\Delta t_{\text {meas. }}^{\prime}=5$ were examined. The optimum estimates of parameters differed from the true values in approximately half of the 100 twin experiments, which yielded large values of $\sigma^{\text {est }}$ under $\Delta t_{\text {meas. }}^{\prime}=5$. The phase diagram calculation and phase-field simulation were performed using an incorrect set of optimum estimates of parameters; the alloy composition was located within the two-phase region in the A-B-C ternary phase diagram, and a phase separation into B-rich and Blean phases was observed in the phase-field simulation (see Supplemental Material Fig. S2 [32]), which is similar to the case shown in Fig. 5 [twin experiment I-(iii)]. Overall, the results of twin experiments I, II, and III indicate that there are some cases where the developed adjoint model fails to estimate parameters depending on the initial guesses of parameters, the time interval of measurements $\Delta t_{\text {meas. }}^{\prime}$ (or the number of measurements $N_{\text {meas. }}$ ), and possibly the magnitude of variance $\sigma^{2}$ in the measurement noise. The validity of 
(a)

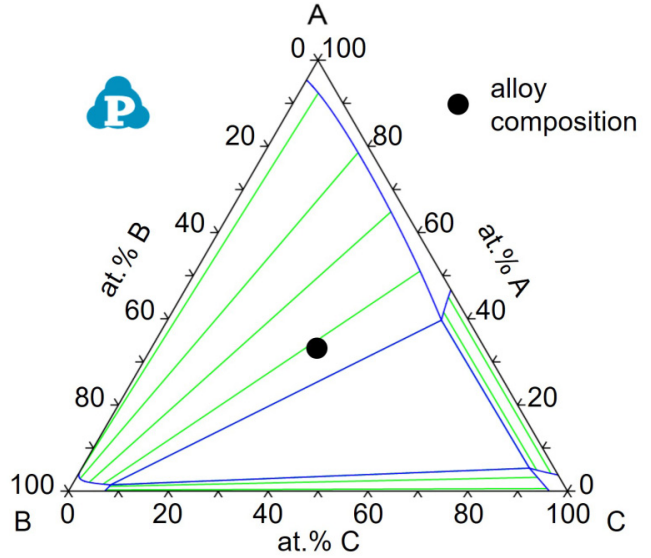

(b)

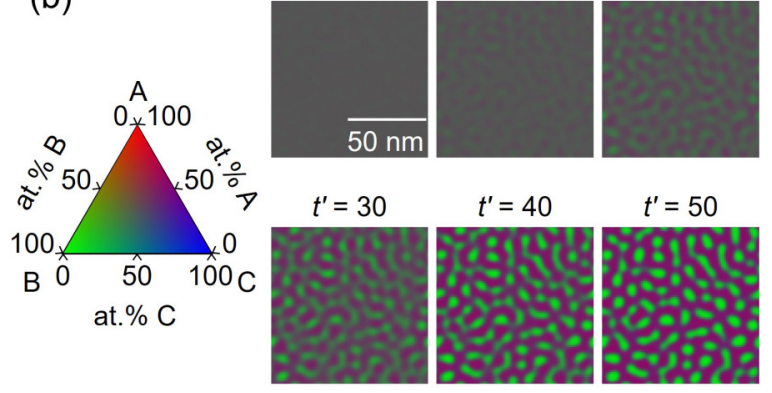

FIG. 5. Calculation result of phase diagram at $900 \mathrm{~K}$ in the A-B-C ternary system (a) and phase-field simulation results of microstructure evolution (b), both of which are obtained using the optimum estimates of parameters in twin experiment I-(iii) (Fig. 4). The ternary color legend is used to show the ternary compositional field in (b). $t^{\prime}$ denotes the dimensionless time in numerical analysis.

the optimum estimates of ${ }^{0} L_{\mathrm{A}, \mathrm{C}},{ }^{1} L_{\mathrm{A}, \mathrm{C}},{ }^{0} L_{\mathrm{B}, \mathrm{C}},{ }^{1} L_{\mathrm{B}, \mathrm{C}}$ should be judged first using the phase diagram calculation. Then, the success or failure of parameter estimation should be evaluated by carefully comparing phase-field simulation using optimum estimates of parameters with time-series measurement data of compositional field maps.

\section{DISCUSSION}

All the twin experiments in Sec. III were performed using the synthetic measurement data shown in Fig. 1(b). We performed an additional twin experiment to confirm the applicability of the developed adjoint model to a synthetic measurement data that is substantially different from the one shown in Fig. 1(b). We assumed an off-centered three-phase region in the A-B-C ternary phase diagram, where the driving force for the spinodal decomposition is small (i.e., the threephase region is small), and hence, the phase separation slowly progresses (see Supplemental Material Fig. S3 [32]). In the additional twin experiment using the newly prepared synthetic measurement data, we confirmed that all optimum estimates of six parameters were coincident with the true values (see Supplemental Material Fig. S4 [32]).

We considered a deterministic dynamical model [Eq. (9)] in this study; given the initial state vector $\mathbf{x}(0)$, Eq. (9) uniquely specifies the trajectory $\{\mathbf{x}(0), \mathbf{x}(1), \mathbf{x}(2), \cdots\}$ of the system. In the estimation of model parameters, we need to find the optimum initial state vector $\hat{\mathbf{x}}(0)$ that minimizes the cost function $J$ [Eq. (11)]. However, because the governing dynamics is nonlinear, it is generally difficult to determine the gradient of $J(\mathbf{x}(0))$. In such a case, the offline DA method (adjoint method) is effective for efficient numerical computation of the gradient of $J(\mathbf{x}(0))$; the computational cost for solving Eq. (14) is comparable to that for solving Eq. (9). The results in Fig. 2 [twin experiment I-(i)] indicate that we can compute the gradient of $J(\mathbf{x}(0))$ by solving Eq. (14), which enables efficient simultaneous estimation of six model parameters of interest. On the contrary, if we consider a stochastic dynamical model, whose randomness is introduced by a random initial state vector or uncertainties in the model, including model errors, online, or sequential DA methods such as the EnKF would be appropriate for estimating model states and parameters. In particular, if the Langevin random fluctuations (stochastic contributions) were incorporated into phase-field equations [33], online DA methods would be useful for estimating model parameters. Another possibility would be to modify the cost function in the adjoint method. Here an additional term should be added to the cost function based on knowledge of the statistical behavior of the Langevin random fluctuations. This type of modification is known as the conversion of a strong constraint problem to a weak constraint problem [9]. The effect of incorporating the Langevin random fluctuations into phase-field equations on the adjoint model and parameter estimation seems critical and thus, can be addressed in the future.

The disadvantage of the adjoint method is that we cannot obtain information about uncertainties of optimum estimates of parameters $\left(\sigma^{\text {est }}\right)$, which are essential information for designing parameter estimation experiments. Ito $e t$ al. assumed that the posterior PDF for parameters of interest can be approximated by a multivariate Gaussian distribution. In that case, $\sigma^{\text {est }}$ can be evaluated from diagonal elements of the inverse of Hessian of $J$, which is obtained through an iterative technique using Hessian-vector products computed on the basis of the second-order adjoint method [14]. However, for the phase-field model described in Sec. II A, we have no idea whether the posterior PDF for six parameters of interest follows a multivariate Gaussian distribution. Hence, we conducted 100 independent tests in twin experiments II and III to evaluate $\sigma^{\text {est }}$. The obtained relation between $\sigma$ and $\sigma^{\text {est }}$ (Fig. 7) or between $\Delta t_{\text {meas. }}^{\prime}$ and $\sigma^{\text {est }}$ (Fig. 9) provides information about necessary experiments to estimate parameters. Conducting at most 100 independent tests in twin experiments seems to be a promising approach to obtaining prior knowledge about $\sigma^{\text {est }}$ at an acceptable computational cost.

The developed adjoint model, which is effective according to twin experiments, should be applied to real experimental data [26] for estimating unknown material parameters. In spinodal decomposition, initial compositional fluctuations significantly affect the subsequent decomposition process and the resultant phase-separated structure. Although it has been assumed that the initial compositional field at $t^{\prime}=0$ is known in advance in our twin experiments, there would be some difficulties in experimentally obtaining compositional field maps 

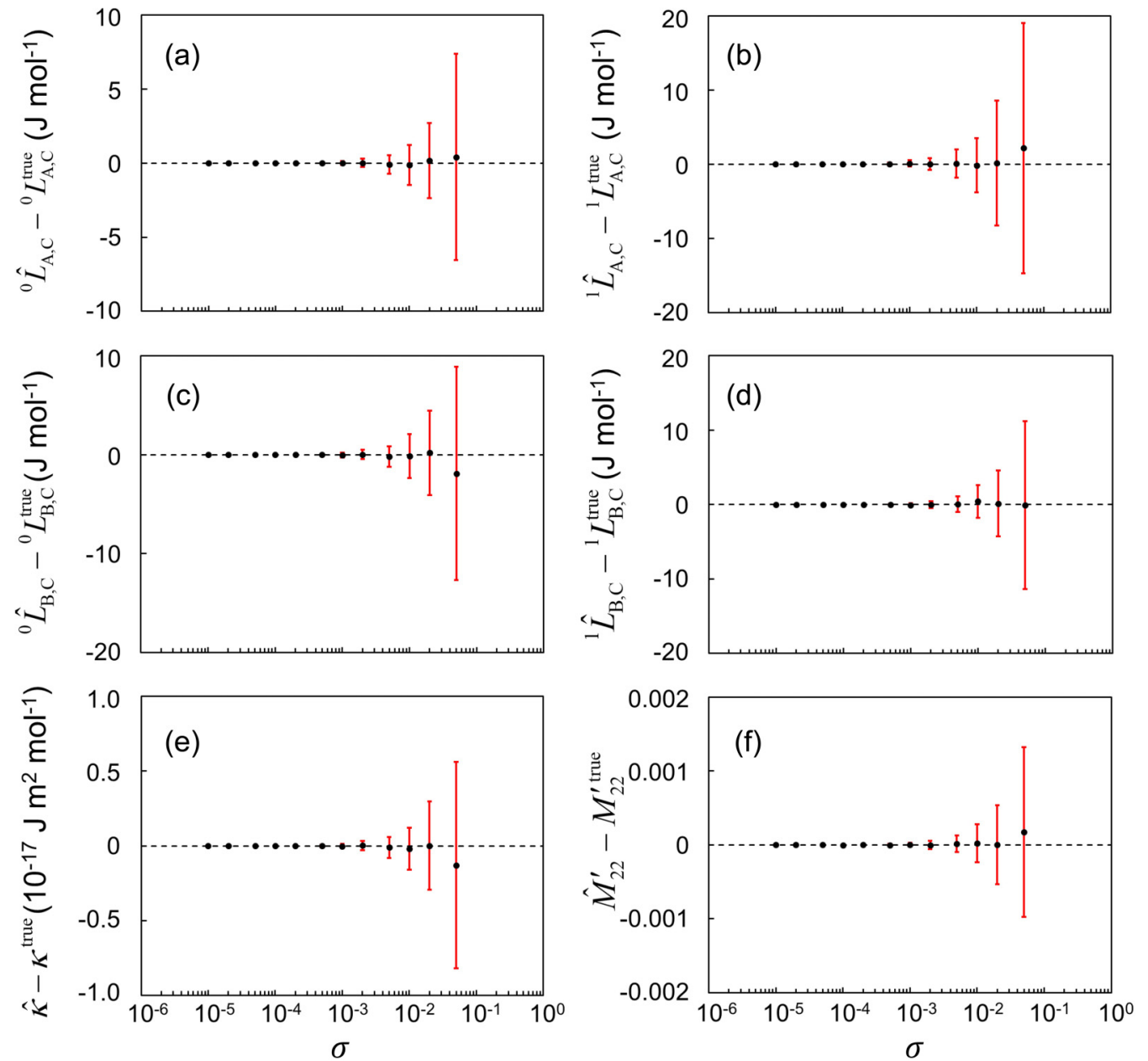

FIG. 6. Results of twin experiment II showing the mean values and standard deviations of optimum estimates of six parameters under various conditions of the standard deviation of measurement noise ( $\sigma$ ): (a) ${ }^{0} L_{\mathrm{A}, \mathrm{C}}$, (b) ${ }^{1} L_{\mathrm{A}, \mathrm{C}}$, (c) ${ }^{0} L_{\mathrm{B}, \mathrm{C}}$, (d) ${ }^{1} L_{\mathrm{B}, \mathrm{C}}$, (e) $\kappa$, and (f) $M_{22}^{\prime}$.

in the initial stage of spinodal decomposition as the signal-tonoise ratio (SNR) is assumed to be low. Hence, in estimating model parameters based on time-series microstructure data, it would also be necessary to estimate unknown compositional field maps in the initial stage of spinodal decomposition using the measurement data obtained in the later stage of spinodal decomposition, where the SNR is assumed to be high. Although we focused on estimating several model parameters in this study, the developed adjoint model has the potential of estimating model states (i.e., initial compositional field) and parameters simultaneously, which should be addressed in the future.

\section{CONCLUSIONS}

An adjoint model was developed to combine a phase-field model for spinodal decomposition in a ternary system with measurement data of time-series compositional field maps, enabling the simultaneous estimation of several unknown parameters in the phase-field model. To evaluate the effectiveness of the developed adjoint model, twin experiments were conducted by varying initial guesses of parameters of interest, the standard deviation of measurement noise $(\sigma)$, and the time interval of measurements $\left(\Delta t_{\text {meas }}^{\prime}\right)$. The obtained results are summarized as follows.

(1) It has been confirmed that the optimum estimates of six model parameters of interest coincide with the true values, indicating that the developed adjoint model is effective for estimating material parameters based on time-series measurement data of microstructure evolution.

(2) Conducting at most 100 twin experiments using 100 synthetic data with different noise terms is a promising approach to evaluating the effect of measurement conditions on the uncertainties of optimum estimates of parameters $\left(\sigma^{\text {est }}\right)$. It has been shown that there is a positive correlation between $\sigma$ and $\sigma^{\text {est }}$ or between $\Delta t_{\text {meas }}^{\prime}$ and $\sigma^{\text {est }}$.

(3) The developed adjoint model failed in estimating model parameters depending on the initial guesses of parameters and measurement conditions of compositional field maps. The validity of the optimum estimates of Gibbs energy parameters should be first judged by calculating the phase diagram. Then, the success or failure of parameter estimation should be evaluated by directly and carefully comparing a phase-field simulation using the optimum estimates of parameters with time-series measurement data of compositional field maps.

The application of the developed adjoint model to real experimental data is expected to contribute significantly to 

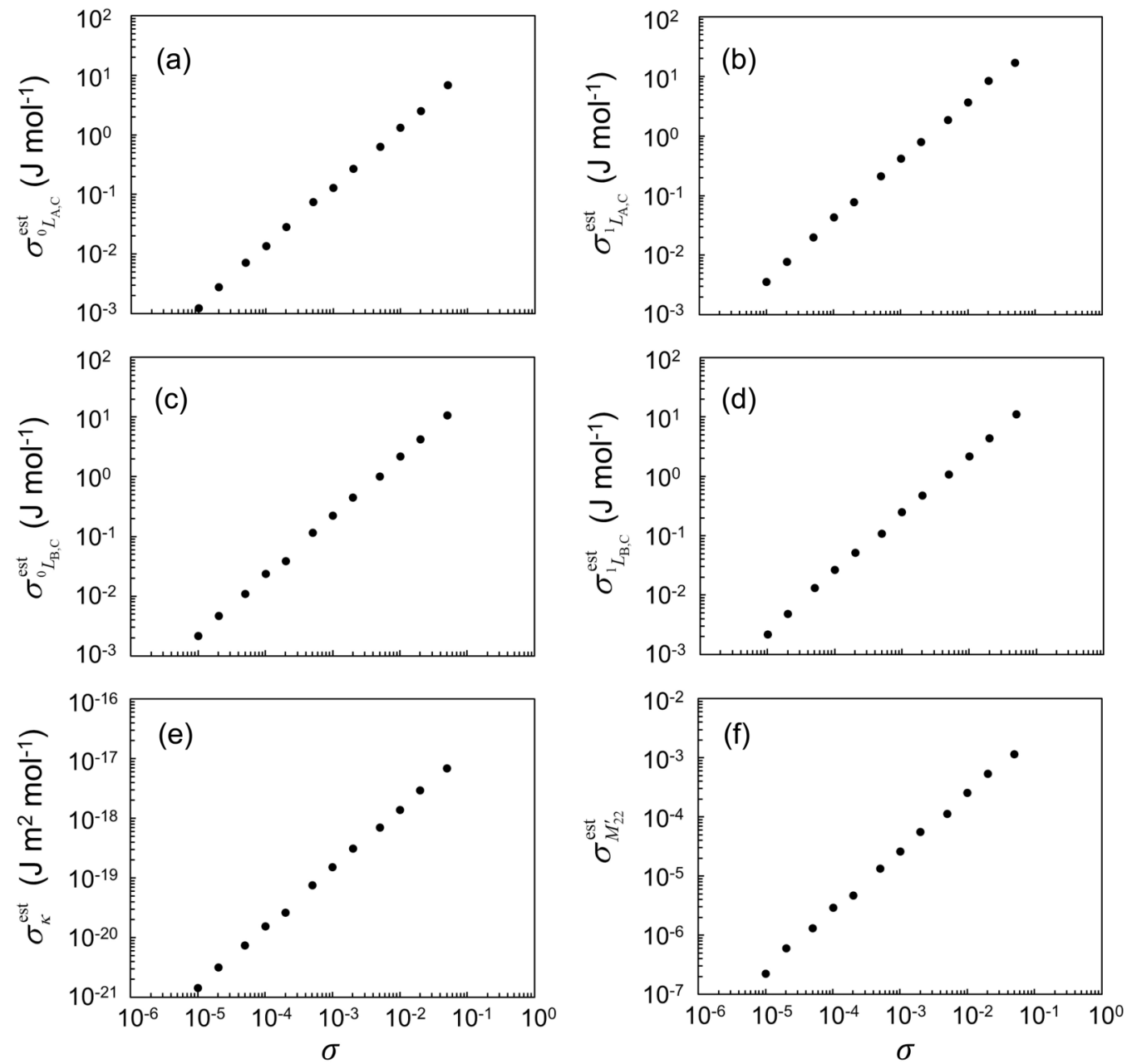

FIG. 7. Results of twin experiment II showing the relation between the standard deviation of measurement noise $(\sigma)$ and the standard deviations of optimum estimates of six parameters $\left(\sigma^{\text {est }}\right.$ ): (a) ${ }^{0} L_{\mathrm{A}, \mathrm{C}},(\mathrm{b}){ }^{1} L_{\mathrm{A}, \mathrm{C}}$, (c) ${ }^{0} L_{\mathrm{B}, \mathrm{C}}$, (d) ${ }^{1} L_{\mathrm{B}, \mathrm{C}},(\mathrm{e}) \kappa$, and (f) $M_{22}^{\prime}$.

the estimation of unknown parameters (Gibbs energy parameters of a nonequilibrium phase, etc.) based on the time-series measurement data of microstructure evolution during spinodal decomposition.

\section{ACKNOWLEDGMENTS}

This work was partially supported by JST PRESTO (Grant No. JPMJPR15NB), JSPS KAKENHI (Grant No. JP21J21292), and MEXT KAKENHI (Grant No. JP18H05454). This work was also partially supported by the Council for Science, Technology and Innovation (CSTI), Cross-ministerial Strategic Innovation Promotion Program (SIP), "Materials Integration for Revolutionary Design System of Structural Materials" (Funding agency: JST). The authors are grateful to Mr. D. Kato (formerly graduate student of Nagoya University, currently at Hitachi Metals, Ltd.) for the collaborative effort to develop the adjoint model. The authors thank Dr. K. Nakazawa (National Institute for Materials Science, Japan) and Dr. T. Mizoguchi (The University of Tokyo, Japan) for fruitful discussions on experimental techniques to obtain time-series compositional field maps during spinodal decomposition.

\section{APPENDIX A: EVOLUTION EQUATIONS OF FIELD VARIABLES}

The Onsager-type phenomenological equations for atomic fluxes in the A-B-C ternary system are expressed as

$$
\begin{aligned}
& \mathbf{J}_{1}=-M_{11} \nabla \mu_{1}-M_{12} \nabla \mu_{2}-M_{13} \nabla \mu_{3}, \\
& \mathbf{J}_{2}=-M_{21} \nabla \mu_{1}-M_{22} \nabla \mu_{2}-M_{23} \nabla \mu_{3}, \\
& \mathbf{J}_{3}=-M_{31} \nabla \mu_{1}-M_{32} \nabla \mu_{2}-M_{33} \nabla \mu_{3},
\end{aligned}
$$

where $M_{i j}$ is the Onsager coefficient. As the total fluxes of atoms are zero,

$$
\mathbf{J}_{1}+\mathbf{J}_{2}+\mathbf{J}_{3}=0 .
$$

Substituting (A1) into (A2), we obtain

$$
\begin{aligned}
& M_{11}+M_{21}+M_{31}=0, \\
& M_{12}+M_{22}+M_{32}=0, \\
& M_{13}+M_{23}+M_{33}=0 .
\end{aligned}
$$

From the Onsager reciprocal relation [34],

$$
\begin{aligned}
& M_{12}=M_{21}, \\
& M_{13}=M_{31}, \\
& M_{23}=M_{32} .
\end{aligned}
$$



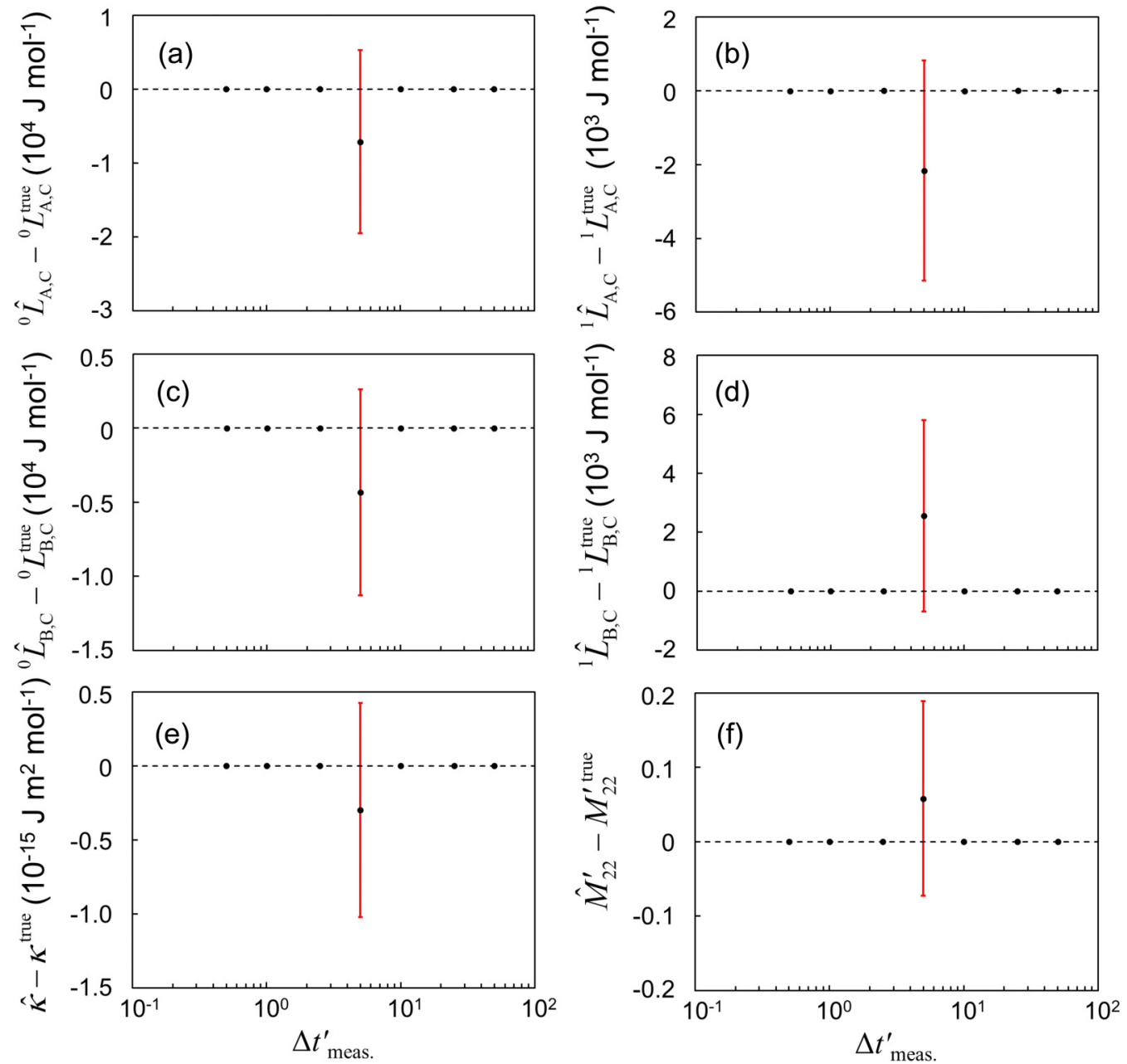

FIG. 8. Results of twin experiment III showing the mean values and standard deviations of optimum estimates of six parameters under various conditions of the time interval of measurements $\left(\Delta t_{\text {meas. }}^{\prime}\right.$ ): (a) ${ }^{0} L_{\mathrm{A}, \mathrm{C}}$, (b) ${ }^{1} L_{\mathrm{A}, \mathrm{C}}$, (c) ${ }^{0} L_{\mathrm{B}, \mathrm{C}}$, (d) ${ }^{1} L_{\mathrm{B}, \mathrm{C}}$, (e) $\kappa$, and (f) $M_{22}^{\prime}$.

Substituting (A3) and (A4)) into (A1), we obtain

$$
\begin{aligned}
\mathbf{J}_{2} & =-M_{21} \nabla \mu_{1}-M_{22} \nabla \mu_{2}-M_{23} \nabla \mu_{3} \\
& =-\left(-M_{22}-M_{23}\right) \nabla \mu_{1}-M_{22} \nabla \mu_{2}-M_{23} \nabla \mu_{3} \\
& =-M_{22}\left(\nabla \mu_{2}-\nabla \mu_{1}\right)-M_{23}\left(\nabla \mu_{3}-\nabla \mu_{1}\right) \\
& =-M_{22} \nabla \frac{\delta G_{\text {sys }}}{\delta c_{2}}-M_{23} \nabla \frac{\delta G_{\text {sys }}}{\delta c_{3}} \\
\mathbf{J}_{3} & =-M_{31} \nabla \mu_{1}-M_{32} \nabla \mu_{2}-M_{33} \nabla \mu_{3} \\
& =-\left(-M_{32}-M_{33}\right) \nabla \mu_{1}-M_{32} \nabla \mu_{2}-M_{33} \nabla \mu_{3} \\
& =-M_{32}\left(\nabla \mu_{2}-\nabla \mu_{1}\right)-M_{33}\left(\nabla \mu_{3}-\nabla \mu_{1}\right) \\
& =-M_{32} \nabla \frac{\delta G_{\text {sys }}}{\delta c_{2}}-M_{33} \nabla \frac{\delta G_{\text {sys }}}{\delta c_{3}} .
\end{aligned}
$$

From the mass conservation equation, we obtain

$$
\begin{aligned}
\frac{\partial c_{2}}{\partial t} & =-\nabla \cdot \mathbf{J}_{2}=\nabla \cdot\left(M_{22} \nabla \frac{\delta G_{\mathrm{sys}}}{\delta c_{2}}+M_{23} \nabla \frac{\delta G_{\mathrm{sys}}}{\delta c_{3}}\right) \\
& \simeq M_{22} \nabla^{2} \frac{\delta G_{\mathrm{sys}}}{\delta c_{2}}+M_{23} \nabla^{2} \frac{\delta G_{\mathrm{sys}}}{\delta c_{3}}, \\
\frac{\partial c_{3}}{\partial t} & =-\nabla \cdot \mathbf{J}_{3} \\
& =\nabla \cdot\left(M_{32} \nabla \frac{\delta G_{\mathrm{sys}}}{\delta c_{2}}+M_{33} \nabla \frac{\delta G_{\mathrm{sys}}}{\delta c_{3}}\right) \\
& \simeq M_{32} \nabla^{2} \frac{\delta G_{\mathrm{sys}}}{\delta c_{2}}+M_{33} \nabla^{2} \frac{\delta G_{\mathrm{sys}}}{\delta c_{3}},
\end{aligned}
$$

where

$$
\begin{aligned}
\frac{\delta G_{\mathrm{sys}}}{\delta c_{2}}= & \left(1-2 c_{2}-c_{3}\right) L_{\mathrm{A}, \mathrm{B}}-c_{3}{ }^{0} L_{\mathrm{A}, \mathrm{C}}+c_{3}\left(2 c_{2}+3 c_{3}-2\right){ }^{1} L_{\mathrm{A}, \mathrm{C}}+c_{3}{ }^{0} L_{\mathrm{B}, \mathrm{C}}+c_{3}\left(2 c_{2}-c_{3}\right){ }^{1} L_{\mathrm{B}, \mathrm{C}} \\
& +\mathrm{R} T\left\{\ln c_{2}-\ln \left(1-c_{2}-c_{3}\right)\right\}-\kappa\left(2 \nabla^{2} c_{2}+\nabla^{2} c_{3}\right) \\
\frac{\delta G_{\mathrm{sys}}}{\delta c_{3}}= & -c_{2} L_{\mathrm{A}, \mathrm{B}}+\left(1-c_{2}-2 c_{3}\right){ }^{0} L_{\mathrm{A}, \mathrm{C}}+\left(6 c_{3}^{2}+6 c_{2} c_{3}-6 c_{3}+c_{2}^{2}-2 c_{2}+1\right){ }^{1} L_{\mathrm{A}, \mathrm{C}}+c_{2}{ }^{0} L_{\mathrm{B}, \mathrm{C}}+c_{2}\left(c_{2}-2 c_{3}\right){ }^{1} L_{\mathrm{B}, \mathrm{C}} \\
& +\mathrm{R} T\left\{\ln c_{3}-\ln \left(1-c_{2}-c_{3}\right)\right\}-\kappa\left(\nabla^{2} c_{2}+2 \nabla^{2} c_{3}\right) .
\end{aligned}
$$



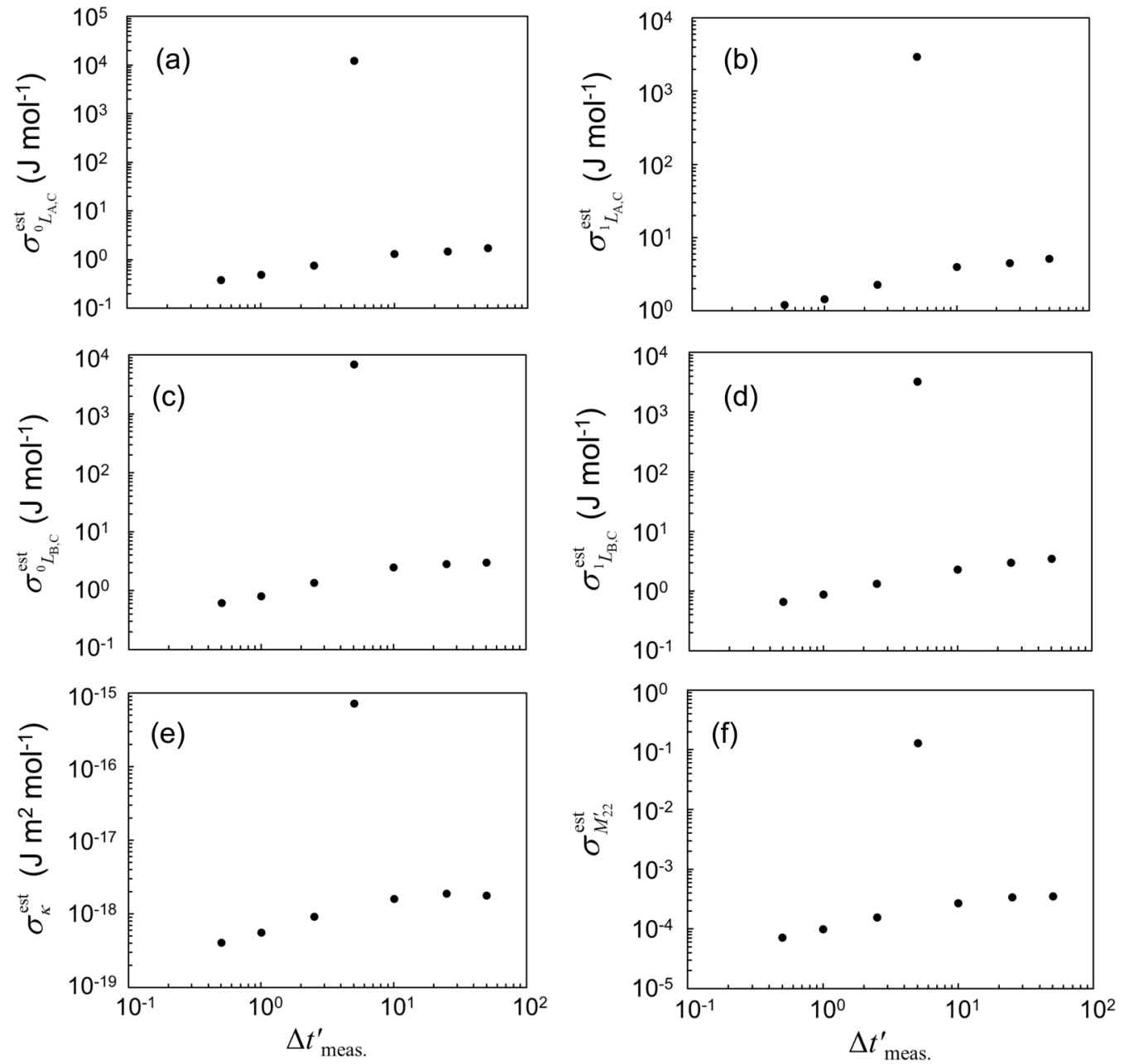

FIG. 9. Results of twin experiment III showing the relation between the time interval of measurements $\left(\Delta t_{\text {meas. }}^{\prime}\right)$ and the standard deviations of optimum estimates of six parameters $\left(\sigma^{\text {est }}\right.$ ): (a) ${ }^{0} L_{\mathrm{A}, \mathrm{C}}$, (b) ${ }^{1} L_{\mathrm{A}, \mathrm{C}}$, (c) ${ }^{0} L_{\mathrm{B}, \mathrm{C}}$, (d) ${ }^{1} L_{\mathrm{B}, \mathrm{C}}$, (e) $\kappa$, and (f) $M_{22}^{\prime}$.

\section{APPENDIX B: ADJOINT MODEL}

The Lagrangian multipliers corresponding to $c_{2}, c_{3},{ }^{0} L_{\mathrm{A}, \mathrm{C}},{ }^{1} L_{\mathrm{A}, \mathrm{C}},{ }^{0} L_{\mathrm{B}, \mathrm{C}},{ }^{1} L_{\mathrm{B}, \mathrm{C}}, \kappa$, and $M_{22}$ are denoted as $\lambda_{c 2}, \lambda_{c 3}, \lambda_{L \mathrm{AC} \_} 0$, $\lambda_{L A C_{-} 1}, \lambda_{L B B_{-} 0}, \lambda_{L B_{-} \_1}, \lambda_{K}$, and $\lambda_{M 22}$, respectively. The adjoint model given by Eq. (14) is expressed in the final form

$$
\begin{gathered}
\frac{\partial \lambda_{c 2}}{\partial t}=-M_{22}\left(\nabla^{2} \lambda_{c 2} \frac{\partial \mu_{2}^{\mathrm{c}}}{\partial c_{2}}-2 \kappa \nabla^{4} \lambda_{c 2}\right)-M_{23}\left(\nabla^{2} \lambda_{c 2} \frac{\partial \mu_{3}^{\mathrm{c}}}{\partial c_{2}}-\kappa \nabla^{4} \lambda_{c 2}\right) \\
-M_{32}\left(\nabla^{2} \lambda_{c 3} \frac{\partial \mu_{2}^{\mathrm{c}}}{\partial c_{2}}-2 \kappa \nabla^{4} \lambda_{c 3}\right)-M_{33}\left(\nabla^{2} \lambda_{c 3} \frac{\partial \mu_{3}^{\mathrm{c}}}{\partial c_{2}}-\kappa \nabla^{4} \lambda_{c 3}\right)-\frac{\partial \mathcal{J}}{\partial c_{2}} \\
\frac{\partial \lambda_{c 3}}{\partial t}=-M_{22}\left(\nabla^{2} \lambda_{c 2} \frac{\partial \mu_{2}^{\mathrm{c}}}{\partial c_{3}}-\kappa \nabla^{4} \lambda_{c 2}\right)-M_{23}\left(\nabla^{2} \lambda_{c 2} \frac{\partial \mu_{3}^{\mathrm{c}}}{\partial c_{3}}-2 \kappa \nabla^{4} \lambda_{c 2}\right) \\
-M_{32}\left(\nabla^{2} \lambda_{c 3} \frac{\partial \mu_{2}^{\mathrm{c}}}{\partial c_{3}}-\kappa \nabla^{4} \lambda_{c 3}\right)-M_{33}\left(\nabla^{2} \lambda_{c 3} \frac{\partial \mu_{3}^{\mathrm{c}}}{\partial c_{3}}-2 \kappa \nabla^{4} \lambda_{c 3}\right)-\frac{\partial \mathcal{J}}{\partial c_{3}} \\
\frac{\partial \lambda_{L \mathrm{AC} \_} 0}{\partial t}=-\int_{\mathbf{r}}\left(M_{22} \nabla^{2} \lambda_{c 2} \frac{\partial \mu_{2}^{\mathrm{c}}}{\partial^{0} L_{\mathrm{A}, \mathrm{C}}}+M_{23} \nabla^{2} \lambda_{c 2} \frac{\partial \mu_{3}^{\mathrm{c}}}{{ }^{0} L_{\mathrm{A}, \mathrm{C}}}+M_{32} \nabla^{2} \lambda_{c 3} \frac{\partial \mu_{2}^{\mathrm{c}}}{\partial^{0} L_{\mathrm{A}, \mathrm{C}}}+M_{33} \nabla^{2} \lambda_{c 3} \frac{\partial \mu_{3}^{\mathrm{c}}}{\partial{ }^{0} L_{\mathrm{A}, \mathrm{C}}}\right) d \mathbf{r} \\
\frac{\partial \lambda_{L \mathrm{AC} \_1}=-}{\partial t}=-\int_{\mathbf{r}}\left(M_{22} \nabla^{2} \lambda_{c 2} \frac{\partial \mu_{2}^{\mathrm{c}}}{\partial^{1} L_{\mathrm{A}, \mathrm{C}}}+M_{23} \nabla^{2} \lambda_{c 2} \frac{\partial \mu_{3}^{\mathrm{c}}}{\partial^{1} L_{\mathrm{A}, \mathrm{C}}}+M_{32} \nabla^{2} \lambda_{c 3} \frac{\partial \mu_{2}^{\mathrm{c}}}{\partial^{1} L_{\mathrm{A}, \mathrm{C}}}+M_{33} \nabla^{2} \lambda_{c 3} \frac{\partial \mu_{3}^{\mathrm{c}}}{\partial{ }^{1} L_{\mathrm{A}, \mathrm{C}}}\right) d \mathbf{r} \\
\frac{\partial \lambda_{L \mathrm{BC} \_0}=-}{\partial t}=-\int_{\mathbf{r}}\left(M_{22} \nabla^{2} \lambda_{c 2} \frac{\partial \mu_{2}^{\mathrm{c}}}{\partial^{0} L_{\mathrm{B}, \mathrm{C}}}+M_{23} \nabla^{2} \lambda_{c 2} \frac{\partial \mu_{3}^{\mathrm{c}}}{\partial^{0} L_{\mathrm{B}, \mathrm{C}}}+M_{32} \nabla^{2} \lambda_{c 3} \frac{\partial \mu_{2}^{\mathrm{c}}}{\partial^{0} L_{\mathrm{B}, \mathrm{C}}}+M_{33} \nabla^{2} \lambda_{c 3} \frac{\partial \mu_{3}^{\mathrm{c}}}{\partial^{0} L_{\mathrm{B}, \mathrm{C}}}\right) d \mathbf{r}
\end{gathered}
$$




$$
\begin{gathered}
\frac{\partial \lambda_{L \mathrm{BC} \_} 1}{\partial t}=-\int_{\mathbf{r}}\left(M_{22} \nabla^{2} \lambda_{c 2} \frac{\partial \mu_{2}^{\mathrm{c}}}{\partial{ }^{1} L_{\mathrm{B}, \mathrm{C}}}+M_{23} \nabla^{2} \lambda_{c 2} \frac{\partial \mu_{3}^{\mathrm{c}}}{\partial{ }^{1} L_{\mathrm{B}, \mathrm{C}}}+M_{32} \nabla^{2} \lambda_{c 3} \frac{\partial \mu_{2}^{\mathrm{c}}}{\partial{ }^{1} L_{\mathrm{B}, \mathrm{C}}}+M_{33} \nabla^{2} \lambda_{c 3} \frac{\partial \mu_{3}^{\mathrm{c}}}{\partial{ }^{1} L_{\mathrm{B}, \mathrm{C}}}\right) d \mathbf{r} \\
\frac{\partial \lambda_{\kappa}}{\partial t}=\int_{\mathbf{r}}\left(2 M_{22} \lambda_{c 2} \nabla^{4} c_{2}+M_{22} \lambda_{c 2} \nabla^{4} c_{3}+M_{23} \lambda_{c 2} \nabla^{4} c_{2}+2 M_{23} \lambda_{c 2} \nabla^{4} c_{3}\right. \\
\left.+2 M_{32} \lambda_{c 3} \nabla^{4} c_{2}+M_{32} \lambda_{c 3} \nabla^{4} c_{3}+M_{33} \lambda_{c 3} \nabla^{4} c_{2}+2 M_{33} \lambda_{c 3} \nabla^{4} c_{3}\right) d \mathbf{r} \\
\frac{\partial \lambda_{M 22}}{\partial t}=-\int_{\mathbf{r}}\left[\lambda_{c 2} \nabla^{2} \mu_{2}^{\mathrm{c}}-\kappa\left(2 \lambda_{c 2} \nabla^{4} c_{2}+\lambda_{c 2} \nabla^{4} c_{3}\right)\right] d \mathbf{r},
\end{gathered}
$$

where

$$
\begin{aligned}
\mu_{2}^{\mathrm{c}}= & \left(1-2 c_{2}-c_{3}\right) L_{\mathrm{A}, \mathrm{B}}-c_{3}{ }^{0} L_{\mathrm{A}, \mathrm{C}} \\
& +c_{3}\left(2 c_{2}+3 c_{3}-2\right){ }^{1} L_{\mathrm{A}, \mathrm{C}}+c_{3}{ }^{0} L_{\mathrm{B}, \mathrm{C}}+c_{3}\left(2 c_{2}-c_{3}\right){ }^{1} L_{\mathrm{B}, \mathrm{C}}+\mathrm{R} T\left\{\ln c_{2}-\ln \left(1-c_{2}-c_{3}\right)\right\} \\
\mu_{3}^{\mathrm{c}}= & -c_{2} L_{\mathrm{A}, \mathrm{B}}+\left(1-c_{2}-2 c_{3}\right){ }^{0} L_{\mathrm{A}, \mathrm{C}} \\
& +\left(6 c_{3}^{2}+6 c_{2} c_{3}-6 c_{3}+c_{2}^{2}-2 c_{2}+1\right)^{1} L_{\mathrm{A}, \mathrm{C}}+c_{2}{ }^{0} L_{\mathrm{B}, \mathrm{C}}+c_{2}\left(c_{2}-2 c_{3}\right){ }^{1} L_{\mathrm{B}, \mathrm{C}}+\mathrm{R} T\left\{\ln c_{3}-\ln \left(1-c_{2}-c_{3}\right)\right\} .
\end{aligned}
$$

[1] L. Q. Chen, Annu. Rev. Mater. Res. 32, 113 (2002).

[2] I. Steinbach, Model. Simul. Mater. Sci. Eng. 17, 073001 (2009).

[3] J. Zhang, S. O. Poulsen, J. W. Gibbs, P. W. Voorhees, and H. F. Poulsen, Acta Mater. 129, 229 (2017).

[4] Y. Tsukada, Y. Beniya, and T. Koyama, J. Alloys Compd. 603, 65 (2014).

[5] Y. Tsukada, S. Takeno, M. Karasuyama, H. Fukuoka, M. Shiga, and T. Koyama, Sci. Rep. 9, 15794 (2019).

[6] S. Takeno, Y. Tsukada, H. Fukuoka, T. Koyama, M. Shiga, and M. Karasuyama, Phys. Rev. Mater. 4, 083802 (2020).

[7] S. Takeno, H. Fukuoka, Y. Tsukada, T. Koyama, M. Shiga, I. Takeuchi, and M. Karasuyama, Proc. Mach. Learn. Res. 119, 9334 (2020).

[8] J. M. Lewis, S. Lakshmivarahan, and S. K. Dhall, Dynamic Data Assimilation: A Least Squares Approach (Cambridge University Press, Cambridge, 2006).

[9] G. Evensen, Data Assimilation: The Ensemble Kalman Filter, 2nd ed. (Springer, Berlin, 2009).

[10] W. Lahoz, B. Khattatov, and R. Ménard, in Data Assimilation: Making Sense of Observations, edited by W. Lahoz, B. Khattatov, and R. Ménard (Springer, Berlin, 2010).

[11] K. Sasaki, A. Yamanaka, S. Ito, and H. Nagao, Comput. Mater. Sci. 141, 141 (2018).

[12] A. Yamanaka, Y. Maeda, and K. Sasaki, Mater. Des. 165, 107577 (2019).

[13] M. Ohno, Y. Oka, S. Sakane, Y. Shibuta, and T. Takaki, Phys. Rev. E 101, 052121 (2020).

[14] S. Ito, H. Nagao, A. Yamanaka, Y. Tsukada, T. Koyama, M. Kano, and J. Inoue, Phys. Rev. E 94, 043307 (2016).

[15] S. Ito, H. Nagao, T. Kasuya, and J. Inoue, Sci. Technol. Adv. Mater. 18, 857 (2017).

[16] T. Miyazaki, S. Takagishi, H. Mori, and T. Kozakai, Acta Metall. 28, 1143 (1980).
[17] T. Kozakai, H. Aihara, M. Doi, and T. Miyazaki, Trans. Iron Steel Inst. Jpn. 25, 159 (1985).

[18] Y. Koyama, Acta Metall. Mater. 38, 857 (1990).

[19] D. Bouttes, E. Gouillart, E. Boller, D. Dalmas, and D. Vandembroucq, Phys. Rev. Lett. 112, 245701 (2014).

[20] B. Ditchek and L. H. Schwartz, Acta Metall. 28, 807 (1980).

[21] K. B. Rundman and J. E. Hilliard, Acta Metall. 15, 1025 (1967).

[22] T. Ujihara and K. Osamura, Acta Mater. 48, 1629 (2000).

[23] J. E. Westraadt, E. J. Olivier, J. H. Neethling, P. Hedström, J. Odqvist, X. Xu, and A. Steuwer, Mater. Charact. 109, 216 (2015).

[24] J. Palisaitis, C. L. Hsiao, L. Hultman, J. Birch, and P. O. Persson, Sci. Rep. 7, 44390 (2017).

[25] K. Nakazawa, T. Miyata, S. Amma, and T. Mizoguchi, Scr. Mater. 154, 197 (2018).

[26] K. Nakazawa, S. Amma, and T. Mizoguchi, Acta Mater. 200, 720 (2020).

[27] N. Saunders and A. P. Miodownik, CALPHAD (Calculation of Phase Diagrams): A Comprehensive Guide (Pergamon, Oxford, 1998).

[28] E. B. Nauman and D. Q. He, Polymer 35, 2243 (1994).

[29] J. W. Cahn and J. E. Hilliard, J. Chem. Phys. 28, 258 (1958).

[30] J. E. Dennis and J. J. Moré, SIAM Rev. 19, 46 (1977).

[31] L. Armijo, Pac. J. Math. 16, 1 (1966).

[32] See Supplemental Material at http://link.aps.org/supplemental/ 10.1103/PhysRevMaterials.5.113801 for twin experiment I-(ii), phase diagram calculation, and phase-field simulation using an incorrect set of optimum estimates of parameters in twin experiment III, and twin experiment using other synthetic measurement data.

[33] R. G. Petschek and H. Metiu, J. Chem. Phys. 79, 3443 (1983).

[34] L. Onsager, Phys. Rev. 37, 405 (1931). 